\title{
TICS NA EDUCAÇÃO: O USO DE SOFTWARE LIVRE NA PROMOÇÃO DA ACESSIBILIDADE
}

Magnólia Maria Medeiros; Maria Jane Queiroz magnolia.maria@academico.ifrn.edu.br; jane.

queiroz@ifrn.edu.br

Instituto Federal de Educação, Ciência e Tecnologia do Rio Grande do Norte

DOI: $10.15628 /$ rbept.2018.6875

Artigo submetido em dez/2017 e aceito em mar/2018

\section{RESUMO}

Este artigo descreve uma pesquisa-ação em desenvolvimento através de um projeto de extensão denominado "TICs na educação: o uso de software livre na promoção da acessibilidade", cuja finalidade é promover a oferta de uma educação verdadeiramente inclusiva no tocante ao uso de tecnologias assistivas como recursos para a inclusão social e participação de alunos com deficiência, mobilidade reduzida ou incapacidade no cotidiano escolar, através da atuação de docentes capacitados no uso de tais tecnologias. Uma das ações do projeto é a oferta de um curso de capacitação em tecnologias assistivas utilizando software livre, a fim de capacitar docentes em sua atuação com alunos deficientes, aprimorando sua prática e possibilitando a inserção do aluno no processo de ensino-aprendizagem. Esta demanda surgiu a partir de visitas a escolas públicas municipais e estaduais da cidade de Currais Novos - Rio Grande do Norte, em que observou-se uma quantidade insuficiente de docentes capacitados para manusear adequadamente os recursos tecnológicos disponíveis, além da existência de laboratórios ociosos e/ou máquinas contendo softwares piratas. Assim, como resultados do projeto, esperase disseminar e incentivar a formação docente para atuação junto aos alunos deficientes; contribuir para esta formação, auxiliando, ainda que indiretamente, a participação, inclusão e aprendizagem de alunos deficientes; estimular o uso do hardware existente nos laboratórios e Salas de Recursos Multifuncionais e combater a pirataria em laboratórios de informática e Salas de Recursos Multifuncionais através do uso de software livre.

Palavras-chave: Acessibilidade, Inclusão Social, Software livre, Tecnologia Assistiva. 


\title{
TICS IN EDUCATION: THE USE OF FREE SOFTWARE IN THE PROMOTION OF ACCESSIBILITY
}

\begin{abstract}
This article describes a research-action under development through an extension project called "TICs in education: the use of free software in the promotion of accessibility", whose purpose is to promote the provision of a truly inclusive education regarding the use of Assistive Technologies as resources for social inclusion and participation of students with disabilities, reduced mobility or incapacity in the daily school life, through the performance of teachers trained in the use of such technologies. One of the actions of the project is the provision of a training course in Assistive Technologies using free software, in order to train teachers in their work with disabled students, improving their practice and enabling the student's insertion in the teaching-learning process. This demand arose from visits to public and municipal public schools in the city of Currais Novos Rio Grande do Norte, where there was an insufficient number of teachers trained to handle the available technological resources adequately, as well as the existence of idle laboratories and / or machines containing pirated software. Thus, as a result of the project, it is hoped to disseminate and encourage teacher training to work with disabled students; contribute to this training, helping, albeit indirectly, the participation, inclusion and learning of disabled students; to stimulate the use of existing hardware in the laboratories and Multifunction Resource Rooms and to combat piracy in computer labs and Multifunctional Resource Rooms through the use of free software.
\end{abstract}

Keywords: Assistive Technology; Social Inclusion; Free software; Accessibility.

\section{INTRODUÇÃO}

Os benefícios decorrentes do uso das tecnologias como ferramentas de ensino-aprendizagem são reais, como sinaliza Souza e Souza (2010, p. 128), “As novas tecnologias ajudarão de forma efetiva o aluno, quando estes estiverem na escola, e nesse momento se sentirão estimulados a buscar e socializar com esses recursos de forma a melhorar seu desempenho escolar", sendo assim se faz necessário adaptá-la a todos os públicos, inovando o ensino com o auxílio da tecnologia, como complementa Zanela $(2007$, p. 26) "[...] um novo sentido no processo de ensinar desde que consideremos todos os recursos tecnológicos disponíveis, que estejam em interação com o ambiente escolar no processo de ensino-aprendizagem".

Entretanto, para que o educador seja capaz de utilizar novas tecnologias como apoio ao seu trabalho, é necessário que o mesmo tenha conhecimentos básicos em informática, pois dessa forma poderá explorar as informações de que necessita para utilizá-las em sua área de 
conhecimento, transformando em recurso metodológico e consequentemente oferecendo um melhor ensino-aprendizagem. Assim a capacitação dos docentes para utilização de computadores no seu dia a dia contribui para um melhoramento no ensino:

“A formação do professor deve prover condições para que ele construa conhecimento sobre as técnicas computacionais, entenda por que e como integrar o computador na sua prática pedagógica e seja capaz de superar barreiras de ordem administrativa e pedagógica. Essa prática possibilita a transição de um sistema fragmentado de ensino para uma abordagem integradora de conteúdo e voltada para a resolução de problemas específicos do interesse de cada aluno. Finalmente, deve-se criar condições para que o professor saiba recontextualizar o aprendizado e a experiência vivida durante a sua formação para a sua realidade de sala de aula compatibilizando as necessidades de seus alunos e os objetivos pedagógicos que se dispõe a atingir". (VALENTE, 1997, p. 14)

Assim, o projeto de extensão denominado "TICs na educação: o uso de software livre na promoção da acessibilidade" foi pensado, desenvolvido e executado na cidade de Currais Novos - Rio Grande do Norte (RN) com a proposta de ofertar um curso de capacitação para os docentes que atuam junto às pessoas com deficiência, como mobilidade reduzida ou baixa visão, a fim de permitir a estas participarem do cotidiano escolar com alguma autonomia e independência.

Escolas estaduais e municipais da cidade e regiões circunvizinhas já dispõem de Salas de Recursos Multifuncionais (SRMF) para atendimento especializado a alunos com necessidades especiais. No entanto, algumas utilizam softwares piratas instalados em plataforma Microsoft Windows devido à dificuldade que os docentes têm em utilizar software livre e gratuito. Além disso, existem poucos softwares para atendimento à variedade de deficiências existentes, conforme relatado pelos profissionais atuantes nestas escolas. Dessa forma, a capacitação de docentes para o uso de softwares livres voltados para pessoas com limitações físicas e/ou psicológicas, além de apresentar um leque de possíveis softwares a serem utilizados com o público-alvo em questão, diminui potencialmente o uso de softwares piratas e/ou a ociosidade de recursos.

\section{DEFICIÊNCIA, UM BREVE RELATO}

Desde os primórdios da civilização, pessoas com deficiência estão presentes na sociedade e, desde então, a postura da mesma em relação a essas pessoas era e ainda é, em menor escala, de segregação, pois segundo Crochík (2006), esse fenômeno é social, mas também há manifestações 
individuais e, em outro momento, complementa ainda Crochík (2011), "os indivíduos com deficiência são desvalorizados por não poderem participar, tal como os outros, da construção e da manutenção da sociedade".

A trajetória do indivíduo com deficiência é marcada por preconceitos, por lutas, pela busca da igualdade de oportunidades, assim como o direito à cidadania. Historicamente, pessoas deficientes carregavam uma imagem de deformação do físico e/ou da mente e no Brasil não foi diferente. Há relatos de crianças que tinham deficiências físicas ou mentais e que foram, em muitos casos, "abandonadas em lugares assediados por bichos que muitas vezes as mutilavam ou matavam" (JANNUZZI, 2004, p. 9). Houve ainda o caso das crianças do Hospital de Neuropsiquiatria Infantil, localizado no município de Oliveira em Minas Gerais, criado em 1924 como hospital psiquiátrico e que mudou seu perfil em 1946, quando passou a receber crianças com qualquer tipo de deficiência física ou mental, sendo em sua maioria, rejeitadas pelas famílias (ARBEX, 2013).

Atualmente, essa forma de pensar e principalmente agir em relação às pessoas com necessidades especiais vem mudando paulatinamente, assim como a postura da sociedade para com essa parcela da população, pois segundo Mazzotta (2011), a pessoa com deficiência passa, ao longo da história, da marginalização para o assistencialismo, deste para a educação, reabilitação, integração social e atualmente para a inclusão social.

No Brasil, isso ficou evidente a partir da aprovação do Estatuto da Pessoa com Deficiência (Lei no 13.146/2015), ou seja, a Lei Brasileira de Inclusão (LBI), resultado de um processo histórico, ou seja, de leis específicas que surgiram ao longo do tempo, outro exemplo é a Lei $n^{\circ} 7.853 / 1989$ que criminaliza o preconceito em relação às pessoas com deficiência e a Lei no 8.213/1991, ou lei de cotas, que se refere à contratação de deficientes nas empresas. Assim, tal estatuto é um conjunto de leis que visam a inclusão da pessoa com deficiência através de medidas de caráter social, ressaltando o último relator dessa lei, o Senador Romário Farias, no dia 6 de julho de 2015, dia em que a LBI foi aprovada:

“[...] a Lei Brasileira de Inclusão inaugura um novo paradigma no país, em que a sociedade através do estatuto irá se preparar para receber a pessoa com deficiência, e não mais, a pessoa com deficiência terá que se adaptar a uma sociedade, que não está apta a recebê-la".

Sendo assim, de acordo com a LBI, a pessoa com deficiência é aquele indivíduo que tem algum tipo de impedimento de longo prazo, de natureza física, mental, intelectual ou sensorial. Vale salientar que o estatuto tem como um objetivo, assegurar os direitos das pessoas com deficiência, equiparando a promoção das oportunidades e garantindo a acessibilidade.

0 grande desafio é colocar tais leis em prática, num país onde 45,6 milhões de pessoas tem algum tipo de deficiência, de acordo com Censo Demográfico de 2010 (IBGE, 2012). 


\section{REALIDADE ESTATÍSTICA}

Os dados do IBGE - Instituto Brasileiro de Geografia e Estatística (IBGE), no censo demográfico de 2010 (IBGE, 2012, p. 73), mostram que 45.606 .048 milhões de pessoas se declararam com pelo menos uma das deficiências pesquisadas (visual, auditiva, motora, mental ou intelectual), o que corresponde a $23,9 \%$ da população brasileira.

Vale ressaltar que no censo demográfico de 2010, as perguntas foram formuladas de maneira a identificar as deficiências visual, auditiva e motora, com seus graus de severidade, através da percepção da população sobre sua dificuldade em enxergar, ouvir e locomover-se, mesmo com o uso de facilitadores (óculos, lentes de contato, aparelho auditivo ou bengala), e a deficiência mental ou intelectual (IBGE, 2012).

Conforme o censo de 2010 (IBGE, 2012), a Região Nordeste é a que concentra os municípios com os maiores percentuais da população brasileira com pelo menos uma das deficiências investigadas, com destaque para o Rio Grande do Norte (RN), onde 12,0\% de seus municípios apresentaram percentual de pessoas com pelo menos uma das deficiências investigadas acima de $35,0 \%$. Dentre as pessoas com algum tipo de deficiência no RN, 6.929 são cegas, 4.879 são surdas, 13.606 não conseguem se locomover, e 52.028 apresentam algum tipo de deficiência mental ou intelectual (TRIBUNA DO NORTE, 2012).

De acordo com Aldemir Freire, chefe da unidade do IBGE no Rio Grande do Norte, estima-se que haja um aumento no percentual de deficientes no estado devido ao fato de que a população está envelhecendo, aumentando assim o percentual de deficiências (TRIBUNA DO NORTE, 2012).

Esse pequeno percurso histórico e dados estatísticos sobre deficiência se fez necessário compreender o porquê de questões sociais e políticas de inclusão, motivando a sociedade a engajar-se em causas, soluções e formas de incluir essas pessoas, de fato, no meio social. Isso se justifica pelo aumento considerável dessa parcela da população que se declara com algum tipo de deficiência, como mostram os dados do censo de 2010.

Uma das formas de incluir tal parcela da população é por meio da educação, através do uso de procedimentos didáticos e metodológicos para melhorar o processo de ensino-aprendizagem e possibilitar a autonomia e independência de deficientes no meio social. Uma das ferramentas que podem e devem ser utilizados com este objetivo são as tecnologias assistivas, abordadas no próximo tópico.

\section{TECNOLOGIA ASSISTIVA}

De acordo com o Comitê de Ajudas Técnicas (CAT) - uma instância de estudos e de proposição de políticas públicas da Secretaria Especial dos Direitos Humanos da Presidência da República (SEDH/PR), o termo Tecnologia Assistiva (TA) trata-se de: 
“[...] uma área do conhecimento, de característica interdisciplinar, que engloba produtos, recursos, metodologias, estratégias, práticas e serviços que objetivam promover a funcionalidade, relacionada à atividade e participação, de pessoas com deficiência, incapacidades ou mobilidade reduzida, visando sua autonomia, independência, qualidade de vida e inclusão social". (CAT, SEDH, 2007)

Em outras palavras, tecnologia assistiva pode ser qualquer hardware, software ou produto integrado que facilite o acesso ao meio à um grupo de pessoas com algum tipo de limitação física ou mental. São exemplos de tecnologia assistiva um software que traduz um texto escrito em português do Brasil para a Língua Brasileira de Sinais (LIBRAS) ou uma plataforma integrada com câmera que permita a um tetraplégico, através dos movimentos dos olhos ou da cabeça, mover o ponteiro de um mouse.

Assim, o objetivo das tecnologias assistivas é proporcionar à pessoa com deficiência maior independência, inclusão social, qualidade de vida, mobilidade, ampla comunicação, facilitar o aprendizado e o trabalho.

As soluções em Tecnologias Assistivas nascem da necessidade de pessoas que possua algum tipo de dificuldade. Conforme Radabaugh (1993), "Para as pessoas sem deficiência, a tecnologia torna as coisas mais fáceis. Para as pessoas com deficiência, a tecnologia torna as coisas possíveis".

Na perspectiva da educação inclusiva, a Tecnologia Assistiva é um recurso utilizado para ampliar ou possibilitar a aplicação de uma atividade necessária e pretendida por uma pessoa com deficiência, ou seja, a Tecnologia Assistiva favorece a participação de um aluno com deficiência, por exemplo, nas diversas atividades do cotidiano escolar, como por exemplo, recursos de acessibilidade ao computador, entre outros. Para além do cotidiano escolar, a Tecnologia Assistiva possibilita que essas pessoas tenham qualidade de vida, facilitando suas atividades diárias como trabalho, mobilidade, comunicação, etc.

No âmbito escolar, as escolas devem oferecer espaços físicos para realização do Atendimento Educacional Especializado (AEE), onde são disponibilizadas Salas de Recursos Multifuncionais (SRMF), que além de possuírem mobiliários, materiais didáticos e pedagógicos, assim como recursos de acessibilidade, oferecem também equipamentos específicos para o atendimento. Neste ponto, o uso do software livre para a promoção da acessibilidade deve ser debatido, já que essas escolas dispõem de salas equipadas com computadores.

0 ensino oferecido neste tipo de atendimento é diferente do ensino escolar e não pode se caracterizar como reforço escolar nem complementação das atividades escolares. São exemplos práticos do AEE o ensino da Língua Brasileira de Sinais (LIBRAS) e do código BRAILLE e, nesse sentido, a inserção de softwares livres, como por exemplo, software de tradução de textos para Libras, ou até mesmo a utilização de uma distribuição Linux voltada à educação 
especial, poderá auxiliar a acessibilidade não só de deficientes visuais, mas de todos os alunos atendidos, pois contém uma gama de softwares educacionais que auxiliam o processo de ensinoaprendizagem de todos os alunos.

No entanto, muitos professores desconhecem as potencialidades do Linux e de softwares livres existentes e destinados ao público em questão. Em muitos casos as escolas até dispõem de computadores, os quais muitas vezes vem com distribuição Linux voltada para educação que acabam por não serem utilizados ou são instalados softwares piratas, devido à dificuldade dos docentes em utilizar os softwares instalados por padrão nos equipamentos cedidos pelo governo.

Com o objetivo de contribuir para a capacitação e melhoria da qualidade do processo de ensino-aprendizagem com pessoas deficientes, além de diminuir a ociosidade e a pirataria de softwares em equipamentos disponíveis nas escolas de Currais Novos - $\mathrm{RN}$, o projeto denominado "TICs na educação: o uso de software livre na promoção da acessibilidade" foi desenvolvido, tendo sua metodologia descrita a seguir.

\section{METODOLOGIA}

A metodologia utilizada para o desenvolvimento do projeto "TICs na educação: o uso de software livre na promoção da acessibilidade" foi organizada em três etapas principais: planejamento, estudo e execução. A fase de planejamento foi iniciada a partir de visitas às escolas municipais e estaduais da cidade de Currais Novos, a fim de se verificar a demanda por capacitação aos profissionais atuantes em salas de AEE. Ainda na fase de planejamento, foram pensados o período de divulgação, inscrição e duração do curso a ser ofertado, bem como os conteúdos a serem ministrados. Para tanto, eram realizadas reuniões semanais para orientação dos membros do projeto (três alunos de graduação e um do ensino médio integrado à curso técnico) por uma coordenadora, discussão e pesquisa quanto à situação dos deficientes no âmbito escolar.

A segunda fase foi composta pelo estudo e pesquisa de softwares livres e gratuitos que atendessem a(s) deficiência(s) constatadas durantes as visitas às escolas da região. A partir deste estudo, foram escolhidos o sistema operacional a ser utilizado, bem como os programas a serem utilizados e até aplicativos presentes em smartphones a serem apresentados aos cursistas.

A terceira e última fase consistiu na execução do curso de capacitação para docentes de escolas públicas municipais e estaduais da cidade de Currais Novos - RN e regiões circunvizinhas. 0 curso foi realizado a partir de aulas expositivas e práticas que aconteciam uma vez por semana, com duração de três horas, durante sete semanas, no IFRN - Campus Currais Novos.

As aulas foram realizadas em laboratórios de informática contendo computadores com o sistema operacional Vinux e softwares livres adicionais (como Orca, Enable Viacam, Suíte 
VLibras, entre outros) de promoção à acessibilidade. Ao final do curso, como forma de avaliação dos conceitos e softwares estudados, os alunos apresentaram projetos que exemplificam a prática do que foi aprendido em sala de aula.

\section{RESULTADOS}

A partir de visitas às escolas públicas do município de Currais Novos-RN, assim como conversas com os profissionais de educação que atuam com pessoas deficientes, os docentes relataram a falta de capacitação para atuar com esse público e, principalmente, a ausência de recursos tecnológicos adequados para um melhor atendimento a essa parcela da população, ficando clara a demanda existente.

As salas onde o atendimento educacional especializado é realizado contam com equipamentos sucateados, softwares piratas ou desatualizados e profissionais com pouco conhecimento para utilizá-los.

A demanda observada inicialmente nas visitas às escolas se comprovou com a quantidade total de inscritos no curso de capacitação: 56 profissionais (entre docentes, profissionais atuantes em salas de $A E E$, enfermeiros e parentes de pessoas deficientes) de diferentes cidades (Currais Novos, Caicó, Lagoa Nova, Jardim do Seridó, Cerro Corá, Campo Redondo, Natal, Frei Martinho-Paraíba) e escolas (Escola Estadual Tristão de Barros, Escola Municipal de Nossa Senhora, Escola Municipal Ausônio Araújo, Escola Municipal Professor Humberto Gama, Escola Estadual Capitão Mor Galvão, Escola Municipal Cipriano Lopes Galvão, Escola Municipal Prof. ${ }^{a}$ Socorro Amaral, Escola Municipal Prof. ${ }^{\circ}$ Salustiano Medeiros, Escola Estadual Dr. Silvio Bezerra de Melo, Escola Estadual Profa Ester Galvão, Escola Estadual Monsenhor Walfredo Gurgel, CAPE - Centro de Aprendizagem e Projetos Educacionais, Escola Municipal Nossa Senhora da Conceição, Escola Municipal João XXIII, Escola Municipal de Ensino Fundamental Francisco Jeronimo de Medeiros, Escola Estadual Manoel Luís de Maria, Unidade Municipal de Educação Infantil Maria de Lourdes Medeiros, Escola Estadual Professora Iracema Brandão de Araújo, Escola Municipal Coronel Rubens Pereira, Escola Municipal Manoel Martiniano de Medeiros, Escola Estadual Berilo Wanderley, Escola Estadual de Ensino Médio Prefeito Aguitonio Dantas) se interessaram e realizaram inscrição.

Embora a frequência durante o período da capacitação não tenha sido a mesma em relação ao número de inscritos, as aulas teóricas e práticas foram realizadas normalmente, contando com a motivação e satisfação dos presentes.

Poucos docentes tinham conhecimento sobre software livre e Linux, mas demonstraram bastante interesse nos conteúdos e práticas apresentados, ficando notório o desconhecimento sobre as possibilidades concretas relacionadas às tecnologias apresentadas.

Durante o curso, foi utilizado como sistema operacional o Vinux, uma distribuição com kernel Linux voltada para deficientes visuais (pessoas que apresentam baixa visão 
ou cegueira) que já vem com o software Orca, específico para esse tipo de deficiência, em sua instalação padrão. Além disso, foram instalados os seguintes softwares livres nesta distribuição: VLibras -- tradutor de textos em português para a Língua Brasileira de Sinais; Enable Viacam -- software que permite controlar os movimentos do mouse a partir de movimentos com realizados com a cabeça, os quais são capturados por uma webcam; etc.

Além de softwares utilizados em computadores e notebooks, foram apresentados alguns softwares disponíveis para instalação e uso em smartphones, como o Hand Talk -- um tradutor de português para Libras.

Dos 56 inscritos no curso, praticamente metade desse número frequentou as aulas e apenas 17 cursistas concluíram a capacitação com a apresentação do projeto final. Embora a quantidade de alunos concluintes não seja tão alto quanto a quantidade de alunos inscritos, para a realidade em que os municípios se encontram e dada a dificuldade de profissionais em se deslocar de seus municípios aos sábados -- alguns deles intercalando o curso de capacitação com uma especialização ou dissertação de mestrado --, este é um número bastante expressivo, capaz de fazer diferença quanto ao uso e disseminação de software livre frente à uma cultura de pirataria e falta de capacitação em escolas públicas.

Independente de números, o mais importante e gratificante ao longo do projeto foi o interesse dos docentes em aplicar o conhecimento adquirido no curso de capacitação em seus locais de trabalho, mesmo aqueles que não atuam diretamente com pessoas deficientes. Ou seja, se mostraram abertos e receptivos a novos conhecimentos e novas tecnologias, a fim de que seus alunos deficientes possam, de fato, se sentir partícipe do ambiente escolar, promovendo assim não só a acessibilidade, mas também a inclusão social dessa parcela da população. É o corpo escolar se adaptando às necessidades dos alunos com deficiência e garantindo assim a equiparação de oportunidades.

\section{CONCLUSÃO}

Pensar em tecnologia assistiva é, de certa forma, pensar em acessibilidade, em inclusão digital, bem como em inclusão social, remetendo à eliminação de barreiras ao "tornar possível" o que para pessoas com necessidades especiais seria impossível; é pensar no próximo; é, de certa forma, humanizar-se.

A capacitação ofertada aos docentes que atuam ou não com pessoas deficientes proporcionou, tanto para cursistas quanto para instrutores, uma valiosa troca de experiências. De um lado, os docentes que, de certa forma, desconheciam o potencial de tecnologias que podem facilitar seu trabalho, e do outro lado, os instrutores do curso (membros do projeto de extensão) que desconheciam a realidade das pessoas com deficiência, pois é muito difícil para quem não possui alguma deficiência se imaginar como tal. Essas pessoas que carregaram, historicamente, uma ideia de incapacidade, de exclusão, de impossibilidades, 
que felizmente vem sendo desconstruído nas últimas décadas.

É preciso repensar a postura dos professores e da escola diante deste quadro, pensar em mais capacitações, em melhores formas de acolher pessoas com necessidades especiais, em formas de como inseri-las no cotidiano escolar e, consequentemente, na vida.

As tecnologias assistivas trazem um leque de possibilidades para pessoas com deficiência. Não se deve limitar a TA ao uso de computadores, Internet e tecnologia. Deve-se, antes de tudo, planejar seu uso como ferramenta de apoio ao desenvolvimento sociocognitivo dos deficientes (PASSERINO; SANTAROSA, 2002, 2003).

Por este motivo, é que se faz necessário pensar e buscar meios para facilitar o acesso dessa parcela da população às Tecnologias Assistivas, incentivando os profissionais que atuam com deficientes a se capacitar; fomentando políticas públicas de orientação para tal; incentivando mais alunos de graduação em pesquisas e projetos para uso das tecnologias da informação e comunicação e das tecnologias assistivas.

\section{REFERÊNCIAS}

ARBEX, Daniela. Holocausto brasileiro. 1. ed. - São Paulo: Geração Editorial, 2013.

BRASIL. Ministério do Planejamento, Desenvolvimento e Gestão. Portal do Software Público

Brasileiro. VLibras - Suíte Vlibras. Disponível em: <https://softwarepublico.gov.br/social/suitevlibras>. Acesso em: 03 jul. 2017.

CAT - Comitê de Ajudas Técnicas. Ata da Reunião VII, de dezembro de 2007 do Comitê de Ajudas Técnicas. Secretaria Especial dos Direitos Humanos da Presidência da República (CORDE/SEDH/ PR), 2007. Disponível em: <http:// http://www.mj.gov.br/corde/comite.asp>. Acesso em: 16 set. 2009.

CROCHÍK, José Leon, coord. Preconceito e Educação Inclusiva - Brasília: SDH/PR, 2011.

CROCHÍK, José Leon. Preconceito, indivíduo e cultura. 3. ed. São Paulo: Robe Editorial, 2006.

ENABLE VIACAM. Download and installation. Disponível em: <http://eviacam.crea-si.com/>. Acesso em: 10 dez. 2017.

GNOME HELP. Leitor de telas Orca. Disponível em: <https://help.gnome.org/users/orca/stable/ index.html.pt_BR>. Acesso em: 20 dez. 2017.

IBGE. Censo demográfico 2010: características gerais da população, religião e pessoas com deficiência. Ministério do Planejamento, Orçamento e Gestão. Rio de Janeiro: Instituto

IBGE. Censo demográfico 2010: características gerais da população, religião e pessoas com 
deficiência. Ministério do Planejamento, Orçamento e Gestão. Rio de Janeiro: Instituto

Brasileiro de Geografia e Estatística, 2012. 211 p. Disponível em: <http://biblioteca.ibge.gov

.br/visualizacao/periodicos/94/cd_2010_religiao_deficiencia.pdf>. Acesso em: 02 jan. 2018.

JANNUZZI, Gilberta S. de M. A educação do deficiente no Brasil: dos primórdios ao início do século XXI. Campinas-SP. Autores Associados, 2004.

MAZZOTTA, Marcos J. S. Educação especial no Brasil: história e políticas públicas. 6. ed. São Paulo: Cortez, 2011.

PASSERINO, L. e SANTAROSA L.M.C. (2002). REDESPECIAL-BRASIL e Universidade Luterana do Brasil, um relato de experiência. Anais. III Congresso Iberoamericano de Informática na Educação Especial 2002 - CIEE 2002. Fortaleza.

—. (2003) Inclusão Digital de Pessoas com necessidades educacionais especiais: EDUKITO. $8^{\circ}$ Taller Internacional de Software Educativo TISE 2003. Santiago do Chile, Chile 24-26 de novembro.

RADABAUGH, Mary Pat. Study on the Financing of Assistive Technology Devices of Services for Individuals with Disabilities - A report to the president and the congress of the United State, National Council on Disability, Março 1993. Disponível em: <http://www.ccclivecaption.com>. Acesso em: 04 dez. 2007.

SOUZA, Isabel Maria Amorim de; SOUZA, Luciana Virgília Amorim de. O USO DA TECNOLOGIA COMO FACILITADORA DA APRENDIZAGEM DO ALUNO NA ESCOLA. Fórum Identidades, Itabaiana, v. 8, n. 4, p.127-142, jul./dez. 2010. Disponível em: < http://200.17.141.110/periodicos/revista_ forum_identidades/revistas/ARQ_FORUM_IND_8>. Acesso em: 15 dez. 2017.

TRIBUNA DO NORTE. RN tem alto índice de deficiência. Disponível em: <http://www.tribunadonorte. com.br/noticia/rn-tem-alto-indice-de-deficiencia/218857>. Acesso em: 26 jan. 2018.

VALENTE, J. A. Visão analítica da Informática na Educação no Brasil: a questão da formação do professor. Revista Brasileira de Informática na Educação. RS: Sociedade Brasileira de Computação, $\mathrm{n}^{\circ} 1$, set. de 1997.

VINUX PROJECT. What is is Vinux?. Disponível em: <http://vinuxproject.org/>. Acesso em: 25 set. 2017.

VINUX. Vinux: Quick Start Guide. Disponível em: <http://www.vinux.org.uk/quick-start-guide.html>. Acesso em: 26 set. 2017.

ZANELA, Mariluci. O Professor e o "laboratório" de informática: navegando nas suas percepções. 43f. Dissertação (Mestrado em Educação). Universidade Federal do Paraná, Curitiba, 2007. (p. 25-27). 